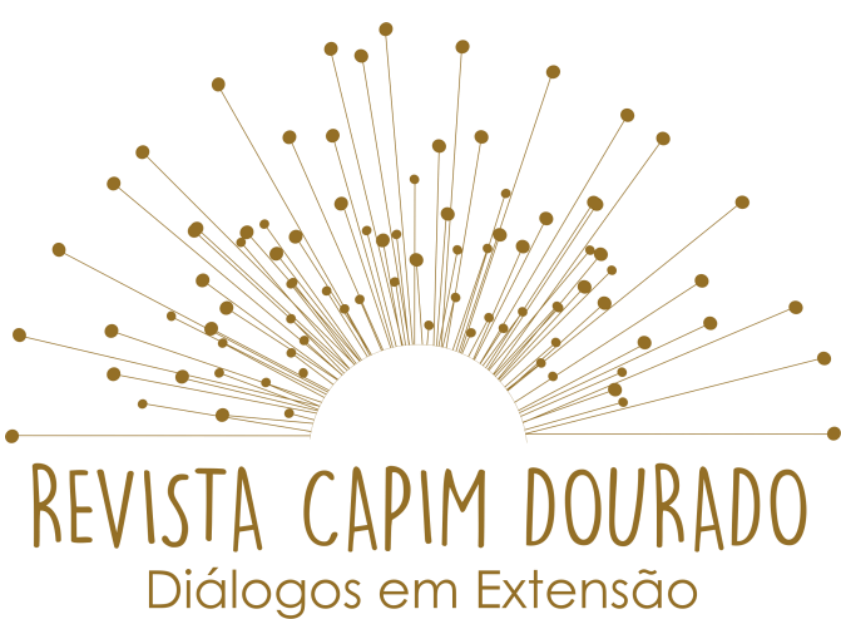

ISSN nำ 2595-7341

Vol. 3, n. 3, Setembro-Dezembro, 2020

\title{
PROJETO ESCUTA SOLIDÁRIA: UNIVERSIDADES NO APOIO ÀS PESSOAS IDOSAS NO CONTEXTO DA COVID-19
}

\author{
SOLIDARY LISTENING PROJECT: UNIVERSITIES SUPPORTING ELDERLY \\ PEOPLE IN THE CONTEXT OF COVID-19
}

PROYECTO DE ESCUCHA SOLIDARIA: UNIVERSIDADES DE APOYO A PERSONAS MAYORES EN EL CONTEXTO DEL COVID-19

\author{
Andréia Queiroz Ribeiro' 1 \\ Maria Sortênia Alves Guimarães ${ }^{2}$ \\ Carolina Araújo dos Santos ${ }^{3}$ \\ Dalila Pinto de Souza Fernandes 4 \\ Daniella Pires Nunes 5 \\ Isabel Cristina Araújo Floriano ${ }^{6}$ \\ Killya de Paiva Santos ${ }^{7}$ \\ Erika Aparecida Bueno 8 \\ Joice da Silva Castro ${ }^{9}$ \\ Jéssica Magalhães Bretas ${ }^{10}$
}

\footnotetext{
1 Doutora em Ciências Farmacêuticas, Professora Associada do Departamento de Nutrição e Saúde da Universidade Federal de Viçosa (UFV), Coordenadora técnico-científica do Programa Municipal da Terceira Idade da UFV/Prefeitura Municipal de Viçosa, Viçosa, MG. E-mail: andreia.ribeiro@ufv.br

${ }^{2}$ Doutora em Ciências da Nutrição, Professora Adjunta do Curso de Medicina da Universidade Federal do Tocantins (UFT), Palmas, TO. E-mail: msortenia@uft.edu.br

${ }^{3}$ Doutora em Ciências da Nutrição, Universidade Federal de Viçosa (UFV), Viçosa, MG. E-mail: carolaraujors@hotmail.com

${ }^{4}$ Doutora em Ciências da Nutrição, Universidade Federal de Viçosa (UFV), Viçosa, MG. E-mail: dalilaf.ufv@gmail.com

5 Doutora em Ciências da Saúde, Docente da Faculdade de Enfermagem da Universidade Estadual de Campinas (Unicamp), Campinas, SP. E-mail: daniellanunes@unicamp.br

${ }^{6}$ Coordenadora do Programa UniversIDADE da Unicamp. E-mail:

isabel.floriano@reitoria.unicamp.br

${ }^{7}$ Mestre em Ciências da Nutrição, Universidade Federal de Viçosa (UFV), Viçosa, MG. E-mail: killya.santos@ufv.br

${ }^{8}$ Coordenadora do Eixo Saúde Física e Mental do Programa UniversIDADE da Unicamp. E-

mail: erika.bueno@reitoria.unicamp.br

${ }^{9}$ Graduanda em Ciências da Nutrição, Universidade Federal de Viçosa (UFV), Viçosa, MG. Email: joice.castro@ufv.br

${ }^{10}$ Graduanda em Ciências da Nutrição, Universidade Federal de Viçosa (UFV), Viçosa, MG. Email: imdejessica@gmail.com
} 


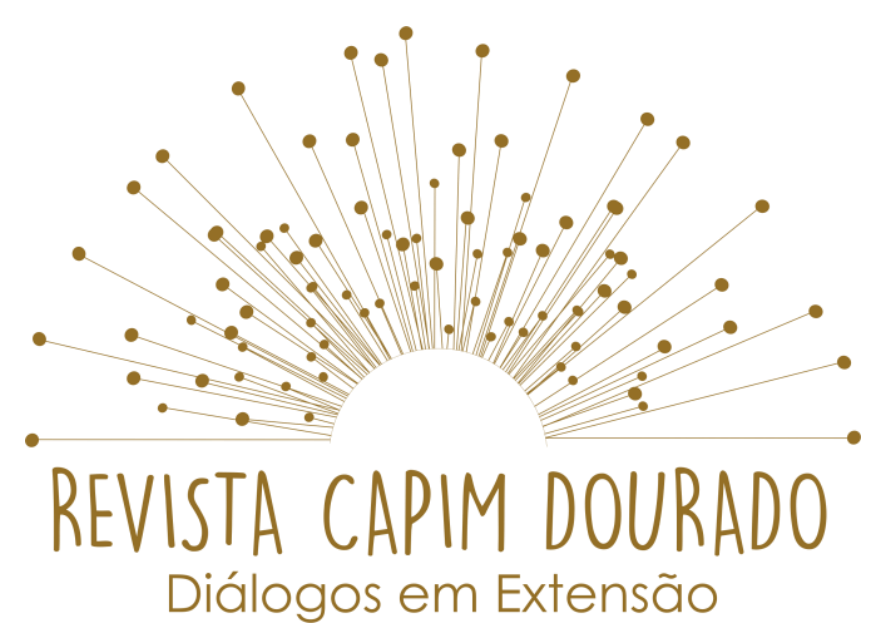

ISSN nำ 2595-7341

Vol. 3, n. 3, Setembro-Dezembro, 2020

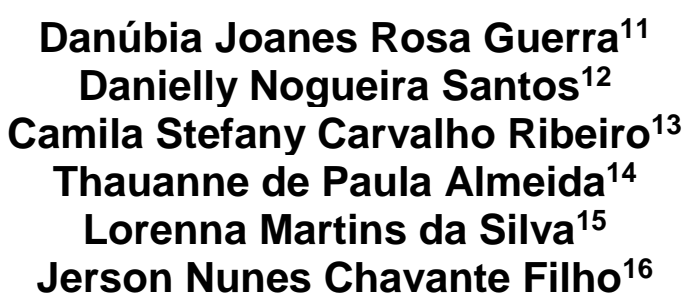

\title{
RESUMO
}

O relato refere-se a uma ação extensionista de escuta solidária a pessoas idosas durante a pandemia da COVID-19. Os idosos são acompanhados por ligações telefônicas, mensagens e ligações pelo Whatsapp, por estudantes universitários, nos municípios de Viçosa (MG), Palmas (TO) e Campinas (SP). A escuta e o acolhimento à pessoa idosa contribuem sobremaneira para fortalecer a autoconfiança, a percepção da transitoriedade do momento, o compartilhamento de enfrentamentos e construção positiva e otimista de que dias melhores virão.

PALAVRAS-CHAVE: Idoso; Solidão; Acolhimento; Pandemia.

\begin{abstract}
The report refers to a solidarity extension action for the older adults during a COVID-19 pandemic. The older adults are accompanied by telephone contacts, messages and contacts via WhatsApp, by university students, in the cities of Viçosa (MG), Palmas (TO) and Campinas (SP). Listening to and welcoming the older adults contributes greatly to strengthening self-confidence, the perception of the transience of the moment, the sharing of confrontations and the positive and optimistic construction of better days.
\end{abstract}

\footnotetext{
${ }^{11}$ Graduanda em Ciências da Nutrição, Universidade Federal de Viçosa (UFV), Viçosa, MG. Email: danubia.guerra@ufv.br

${ }^{12}$ Graduanda em Ciências da Nutrição, Universidade Federal de Viçosa (UFV), Viçosa, MG. Email: danielly.santos@ufv.br

${ }^{13}$ Graduanda em Ciências da Nutrição, Universidade Federal de Viçosa (UFV), Viçosa, MG. Email: camilacarvalho.nutricao@gmail.com

${ }_{14}$ Graduanda em Medicina, Universidade Federal do Tocantins (UFT), Palmas, TO. E-mail: thauannepalmeida@gmail.com

${ }_{15}$ Nutricionista, Coordenadora do Núcleo Ampliado de Saúde da Família e Atenção Básica (NASF-AB), Palmas, TO. E-mail: nutrilorenna.martins@gmail.com

${ }^{16}$ Graduando em Medicina, Universidade Federal do Tocantins (UFT), Palmas, TO. E-mail: jerson nunes@hotmail.com
} 


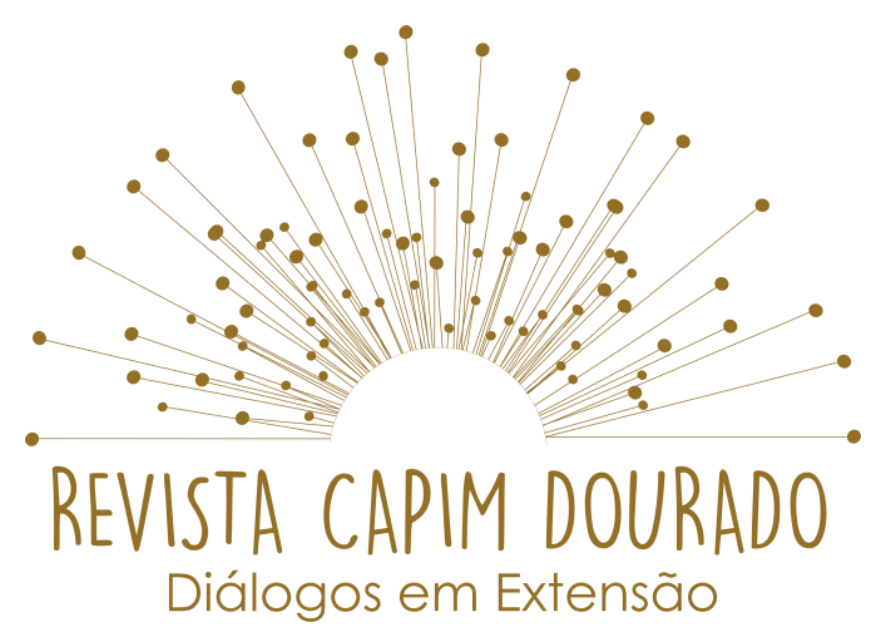

ISSN nำ 2595-7341

Vol. 3, n. 3, Setembro-Dezembro, 2020

KEYWORDS: Aged; Loneliness; User Embracement; Pandemics.

\section{RESUMEN}

El informe se refiere a una acción de extensión solidaria para los ancianos durante una pandemia de COVID-19. Los ancianos están acompañados por contactos telefónicos, mensajes y contactos a través de WhatsApp, por estudiantes universitarios, en los municipios de Viçosa (MG), Palmas (TO) y Campinas (SP). Escuchar y dar la bienvenida a los ancianos contribuye en gran medida a fortalecer la autoconfianza, la percepción de la fugacidad del momento, el intercambio de confrontaciones y la construcción positiva y optimista de días mejores.

PALABRAS CLAVE: Anciano; Soledad; Acogimiento; Pandemia.

\section{A PANDEMIA E SUAS CONSEQUÊNCIAS PARA O COTIDIANO DA PESSOA IDOSA}

As medidas emergenciais e necessárias adotadas pela administração pública dos municípios brasileiros, em consonância com as medidas recomendadas pelas autoridades sanitárias nacionais e internacionais, implicam na necessidade de distanciamento social das pessoas idosas, no intuito de protegê-las da contaminação pelo novo coronavírus (SARS-CoV-2), visto que estão entre os mais vulneráveis à contaminação e às consequências negativas da doença (ARGENTA et al., 2020).

Para as pessoas idosas, a convivência pode ser oportunidade de prosseguimento da vida em coletividade e fator de redução de riscos e vulnerabilidades, contribuindo, desse modo, para a manutenção da autonomia, 


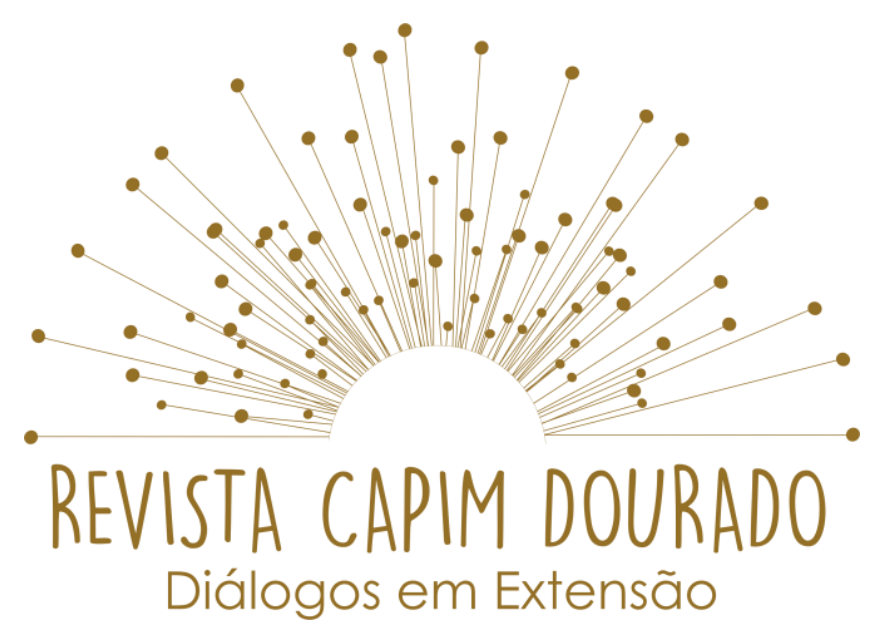

ISSN nำ 2595-7341

Vol. 3, n. 3, Setembro-Dezembro, 2020

da independência e da melhoria de vida durante a velhice (COSTA; SANTOS, 2018). Diante dessa realidade, é sabido que o distanciamento pode desencadear problemas de ordem fisiológica, emocional e social neste momento pandêmico, sendo também a população idosa a mais vulnerável à apresentação desses problemas (WHO, 2020).

Foi neste cenário que grupos de pesquisa e práticas extensionistas de três universidades brasileiras criaram o projeto "Escuta solidária: apoio às pessoas idosas no contexto da COVID-19". Em função da suspensão das atividades presenciais, esses grupos têm se mobilizado para oferecer escuta, acolhimento e orientação aos idosos neste período. Com essa ação, reforça a importância da extensão no apoio à comunidade e contribui para o relevante papel social da universidade no enfrentamento dos desafios sanitários e sociais que o momento atual impõe.

\section{PROPOSTA METODOLÓGICA}

A presente ação extensionista tem sido conduzida sob coordenação das seguintes instituições: Universidade Federal de Viçosa (UFV), Universidade Federal do Tocantins (UFT) e Universidade Estadual de Campinas (UNICAMP). Em cada instituição, um docente é coordenador e nos três locais a equipe conta com a participação de psicólogo.

O projeto acompanha idosos não institucionalizados, atendidos em programas/projetos de extensão, bem como acompanhados pela Estratégia Saúde da Família. Em Palmas, a seleção dos idosos ocorreu por meio da base cadastral da Secretaria Municipal de Saúde; em Campinas, foi implementado no 


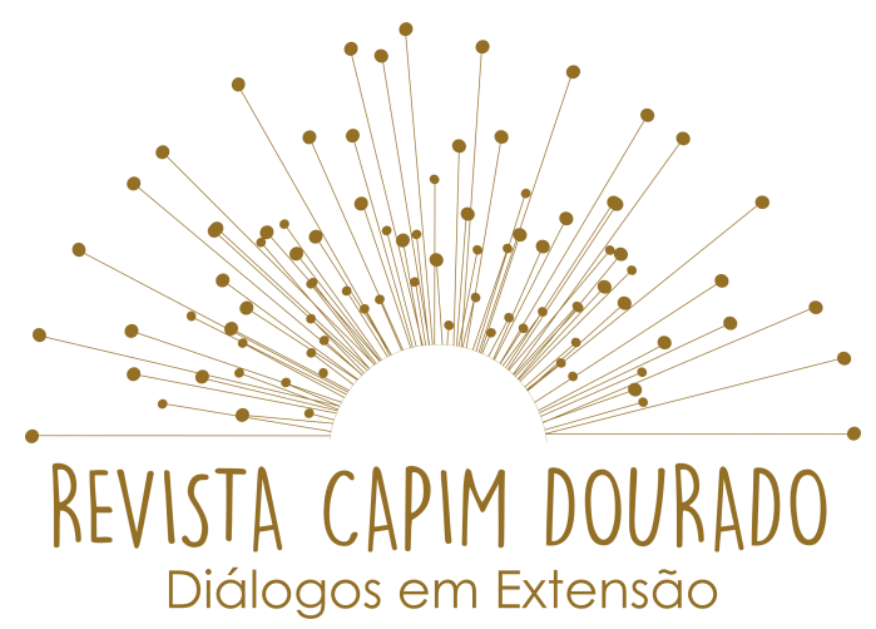

ISSN nำ 2595-7341

Vol. 3, n. 3, Setembro-Dezembro, 2020

Programa UniversIDADE da Unicamp. Em Viçosa, a seleção se deu entre idosos acompanhados pelo Programa Municipal da Terceira Idade e idosos de outros projetos de extensão da UFV.

Foram convidados a participar como voluntários estudantes matriculados em cursos de graduação ou pós-graduação das referidas universidades e que tinham disponibilidade para realizar as ligações por WhatsApp ou telefone. Uma vez selecionados, os voluntários foram treinados virtualmente para a realização do contato. Disponibilizou-se um manual do voluntário, com informações sobre a realização do contato, postura e acolhimento, informações essenciais a serem disponibilizadas para a pessoa idosa, além de orientações para situações delicadas e desafiadoras (como ocorrência de óbito de algum idoso acompanhado).

Durante a realização das ligações telefônicas, o voluntário preenchia um formulário simples, com informações sobre: sexo, idade e escolaridade do idoso; condição do isolamento (se acompanhado ou sozinho); como tem se sentido nesse momento em que precisa ficar em casa; conhecimento sobre as medidas de prevenção da doença; relato de alguma dificuldade nesse período; relato de atividades que o idoso tem realizado no momento de distanciamento; dúvidas que o idoso tem em relação à COVID-19.

Os idosos são acompanhados semanalmente ou de acordo com a necessidade e disponibilidade dos mesmos.

\section{PRINCIPAIS RESULTADOS E AÇÕES}




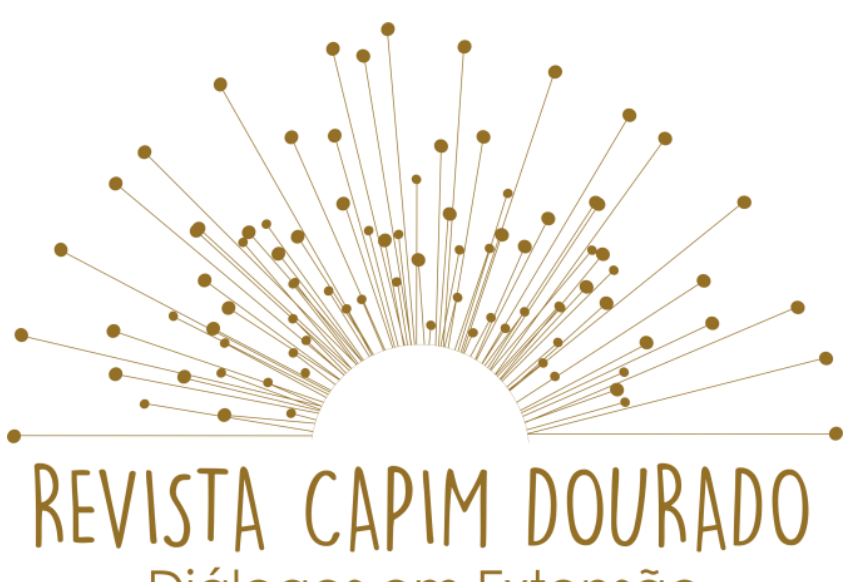

Diálogos em Extensão

Vol. 3, n. 3, Setembro-Dezembro, 2020

O projeto iniciou em abril deste ano na UFV e, posteriormente, estendeuse para UFT e UNICAMP. Até o momento, foram acompanhados 61, 122 e 34 idosos em cada uma dessas instituições, respectivamente. A maioria dos acompanhados são mulheres, com idade média em torno de 69 anos e próximo de $1 / 5$ deles se encontram em isolamento sozinhos (Quadro 1).

Quadro 1. Perfil das pessoas idosas acompanhadas nas instituições, de abril a junho de 2020.

\begin{tabular}{|c|c|c|c|c|}
\hline Instituição & $\mathbf{n}$ & $\begin{array}{c}\mathbf{n}(\%) \text { de } \\
\text { mulheres }\end{array}$ & Idade & Arranjo familiar \\
\hline $\begin{array}{l}\text { Universidade } \\
\text { Federal de } \\
\text { Viçosa }\end{array}$ & 61 & $54(88,5 \%)$ & $\begin{array}{c}\text { De } 54 \text { a } 83 \text { anos } \\
\text { Média: } 67,8 \text { anos } \\
\text { DP: } 6,4 \text { anos }\end{array}$ & $\begin{array}{l}\text { Sozinho:11 }(18,0 \%) \\
\text { Cônjuge: } 5(6,6 \%) \\
\text { Outros familiares/ } \\
\text { amigos: } 45(75,4 \%)\end{array}$ \\
\hline $\begin{array}{l}\text { Universidade } \\
\text { Federal do } \\
\text { Tocantins }\end{array}$ & 122 & $77(63,1 \%)$ & $\begin{array}{c}\text { De } 52 \text { a } 92 \text { anos } \\
\text { Média: } 69,8 \text { anos } \\
\text { DP: } 6,3 \text { anos }\end{array}$ & $\begin{array}{l}\text { Sozinho: } 27(22,1 \%) \\
\text { Cônjuge: } 27(22,1 \%) \\
\text { Outros familiares/ } \\
\text { amigos: } 68(55,8 \%)\end{array}$ \\
\hline $\begin{array}{l}\text { Universidade } \\
\text { Estadual de } \\
\text { Campinas }\end{array}$ & 34 & $23(67,6 \%)$ & $\begin{array}{c}\text { De } 60 \text { a } 79 \text { anos } \\
\text { Média: } 70,1 \text { anos } \\
\text { DP: } 5,6 \text { anos }\end{array}$ & $\begin{array}{l}\text { Sozinho: } 7(20,6 \%) \\
\text { Cônjuge: } 14(41,2 \%) \\
\text { Outros familiares/ } \\
\text { amigos: } 13(38,2 \%)\end{array}$ \\
\hline
\end{tabular}

Houve pouca resistência dos idosos ao primeiro contato e, dentre os que foram resistentes, a maior parte estava em isolamento na companhia de familiares. Entretanto, a regularidade na estratégia de contato, por parte dos voluntários, concorreu para o alcance de boa aceitação e resultou, ainda, na indicação, pelos próprios idosos, de outras pessoas idosas que poderiam se beneficiar com o projeto. 


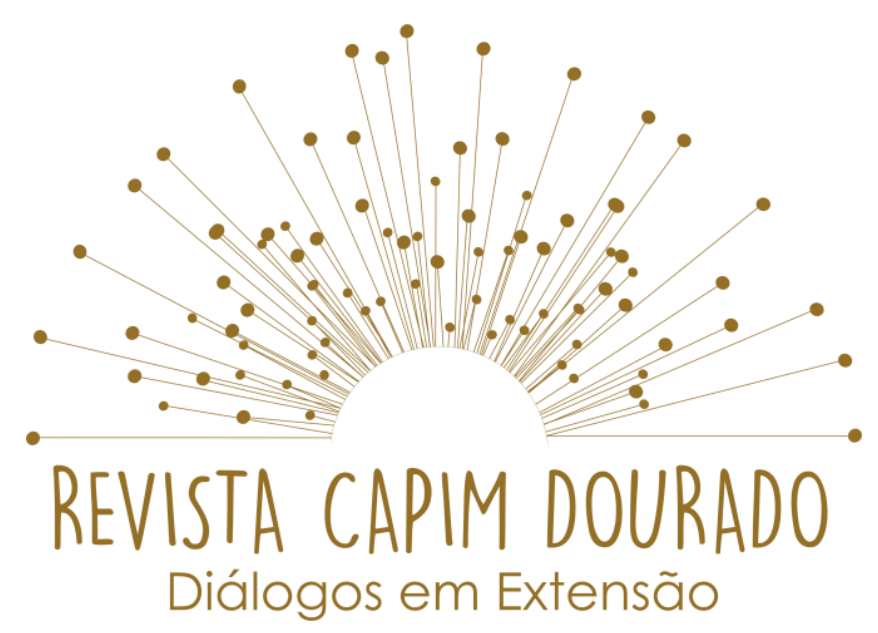

ISSN nำ 2595-7341

Vol. 3, n. 3, Setembro-Dezembro, 2020

De maneira geral, a maioria dos idosos relatou bom grau de conhecimento acerca das medidas de prevenção da COVID e boa percepção acerca da necessidade do distanciamento social.

As principais demandas de orientações apresentadas se referiam a dúvidas sobre a vacinação contra a influenza e retorno dos atendimentos médicos presenciais. Outras demandas, como apoio para acesso a produtos alimentícios e medicamentos foram raras, visto que eles relatavam auxílio de algum vizinho, familiar ou serviço de delivery.

Ao serem perguntados sobre como se sentiam nesse momento, uma proporção importante (mais da metade dos idosos em cada projeto) relatou ansiedade. Houve ainda relato de medo e solidão, os quais resultaram na necessidade de encaminhamento para suporte psicológico, sendo que no município de Viçosa foi solicitada a criação de um serviço de plantão psicológico remoto, para atendimento às pessoas idosas.

A criação de vínculo com os voluntários ocorreu de maneira gradativa. Ao serem perguntados sobre o sentimento de receberem os contatos telefônicos e o acompanhamento pelos voluntários, a grande maioria relatou gratidão e o fato de se sentir importante, conforme se evidencia nos relatos:

"Te desejo muitas felicidades. Sabe porque? Porque você é muito especial para mim. É difícil encontrar parceria com outras pessoas como você me ouça com carinho dedicação e amor. Você me faz a me tornar forte para aceitar as piores coisas da vida" (mensagem de texto no Whatsapp - M, sexo feminino, 68 anos, Viçosa).

"Deus abençoe a sua preocupação, é poucas pessoas que preocupam comigo sabe (...) eu fico muito feliz por isso, fico até emocionada" (mensagem de áudio no Whatsapp - L, sexo feminino, 69 anos, Viçosa).

"Seu contato com a gente, é um anjo do céu que veio pra nós socorrer viu. Você dá muita tranquilidade pra gente. Só de vocês conversar com a gente e 


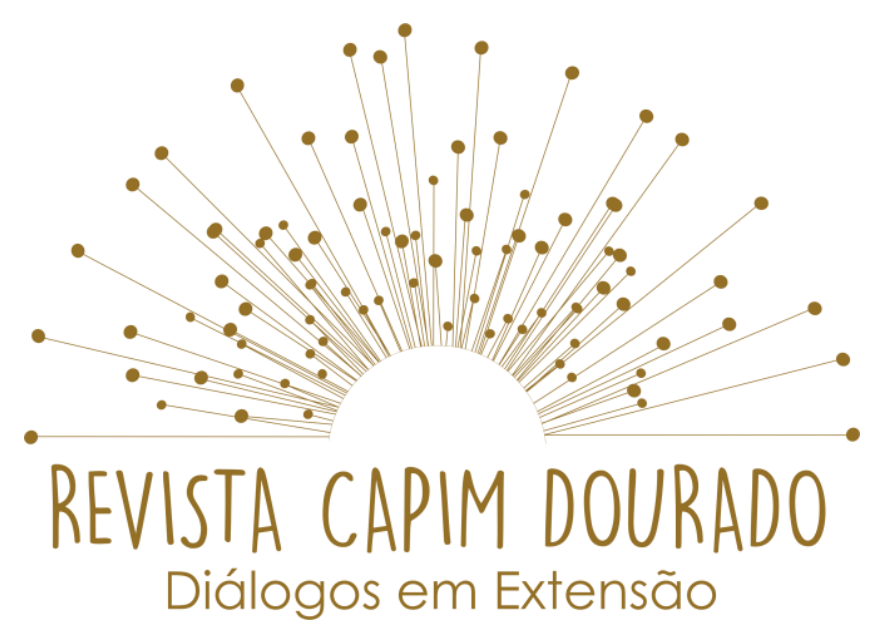

ISSN nำ 2595-7341

Vol. 3, n. 3, Setembro-Dezembro, 2020

dar atenção pra gente, está ajudando a gente demais. A gente sabe que pode apoiar em alguém se tiver algum problema" (mensagem de áudio no Whatsapp - $M$, sexo feminino, 71 anos, Viçosa).

No município de Palmas, desde o início do projeto houve engajamento das equipes da Estratégia Saúde da Família, propiciando o acompanhamento de um maior número de idosos e com maior resolutividade no atendimento às demandas apresentadas. Também em Palmas, o projeto se estendeu para oferecer apoio emocional aos cuidadores de idosos. Já nos municípios de Campinas e Viçosa, não houve envolvimento direto da atenção primária à saúde. A partir da identificação de necessidade dos idosos (realização de consultas, aquisição de medicamentos, realização de exames, acesso a benefícios sociais), algum profissional ou equipamento é acionado, sendo as respostas bastante positivas.

\section{CONSIDERAÇÕES FINAIS}

A experiência aqui apresentada revela a importância do papel social das universidades no estado brasileiro, marcado por profunda desigualdade e falta de serviços e equipamentos básicos para a população. Nesse contexto, a extensão universitária se apresenta como alternativa à promoção de saúde e inserção social da população idosa, fortalecimento de vínculos e oportunidades de aprendizagem e aperfeiçoamento.

A pandemia do novo coronovírus descortinou um conjunto de fragilidades no Brasil relacionadas ao cuidado com as pessoas idosas. A inexistência de uma Política de Cuidados se configura em importante obstáculo para que os idosos 


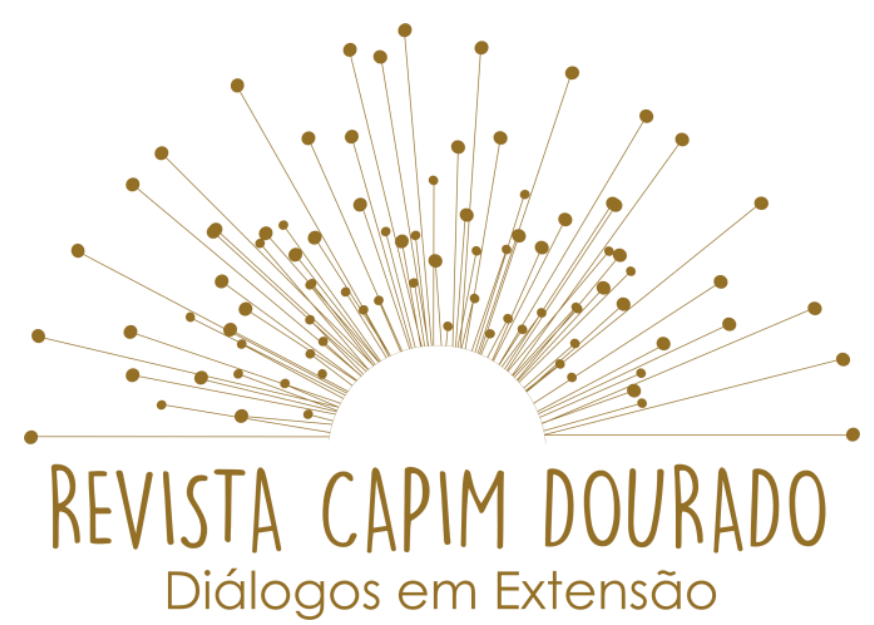

ISSN nำ 2595-7341

Vol. 3, n. 3, Setembro-Dezembro, 2020

tenham condições de adotarem as medidas de distanciamento social em um contexto de suporte físico, social e emocional para o adequado enfrentamento dos impactos do distanciamento e da doença. Nesse sentido, o percurso trilhado pelo presente projeto mostra a importância de uma rede de proteção às pessoas idosas.

\section{REFERÊNCIAS}

ARGENTA, C. et al. Distanciamento social do idoso saudável durante a pandemia COVID-19: possibilidades e desafios. In: ASSOCIAÇÃO BRASILEIRA DE ENFERMAGEM. Enfermagem gerontológica no cuidado do idoso em tempo da COVID 19. Brasília, DF: ABen/DCEG, 2020. Pág. 5-11. Disponível em: <http://www.abennacional.org.br/site/wp-content/uploads/2020/05/E-BOOKGERONTO.pdf>. Acesso em: 26 jun. 2020.

COSTA, S.M.M.; SANTOS, N.B. A vida na velhice. In: MARTINS, S.; RIBEIRO, A.Q. (Org). Envelhecimento ativo: das ações à política. Viçosa: IPPDS, 2018. Pág. 19-27. Disponível em: < http://www.posnutricao.ufv.br/wpcontent/uploads/2019/08/Ebook-envelhecimento-ativo-final.pdf>. Acesso em: 27 jun.2020.

WHO. Mental health and psychosocial considerations during the COVID-19 outbreak. Disponível em: <https://www.who.int/docs/defaultsource/coronaviruse/mental-health-considerations.pdf?sfvrsn=6d3578af_8>. Acesso em: 29 abr. 2020. 